\title{
Food and Fitness: Myths and Truths ${ }^{1}$
}

Linda B. Bobroff ${ }^{2}$

\section{Why the Concern?}

Mass media today is filled with information about nutrition, fitness, and health. Much of the information is inconsistent, and active people may be confused about their nutritional needs. For example, you may wonder if you should take supplements, or if it's okay

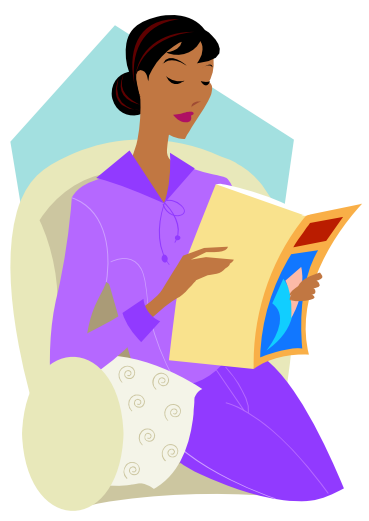
to eat before exercising. This fact sheet will help you make good choices for your health and fitness.

\section{Eating for Fitness}

A healthful eating plan for an active person provides enough protein, fat, carbohydrate, vitamins, minerals, and water to enable the body to work at its optimal level.

Except for energy (calories) and water, nutritional needs are basically the same for people who exercise for fun and health, for athletes, and for those who are less active. We all can use MyPyramid (see Figure 1, page 3) as a guide to eating well for fitness and health.
The amount of food we need varies depending on our age, size, and activity level.

In the quest to be winners, some people fall for diet schemes and supplement claims. There are many popular myths about what active people should eat and drink to get the winning edge. Take this quiz to check your food and fitness IQ.

\section{Food and Fitness Quiz}

For each statement, circle "Myth" or "Truth." Then check your answers and see how you did!

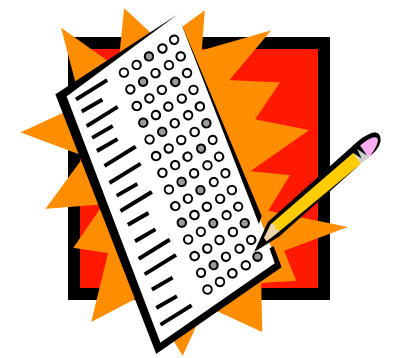

1. Athletes and other active people need protein or amino acid supplements.

Myth Truth

2. A high-carbohydrate diet is best for top performance.

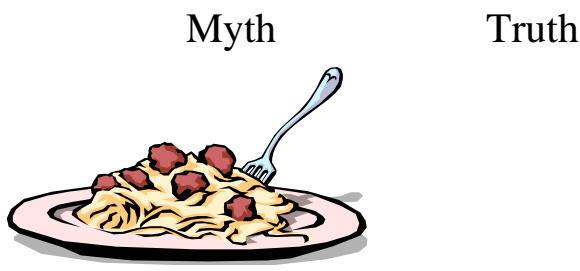

1. This document is FCS8100, one of a series of the Department of Family, Youth and Community Sciences, Florida Cooperative Extension Service, Institute of Food and Agricultural Sciences, University of Florida. First published: July 1986. Revised: April 2006. Please visit the EDIS Web site at http://edis.ifas.ufl.edu

2. Linda B. Bobroff, PhD, RD, LD/N, professor, Department of Family, Youth and Community Sciences, Cooperative Extension Service, Institute of Food and Agricultural Sciences, University of Florida, Gainesville, 32611. 
3. Physically active people who eat a balanced diet need vitamin supplements.

$$
\text { Myth Truth }
$$

4. Chromium picolinate is a safe supplement that boosts performance.

$$
\text { Myth Truth }
$$

5. Drinking water or other fluids during exercise impairs performance.

$$
\text { Myth Truth }
$$

6. A sports drink is a better fluid replacement than water during intense exercise lasting more than one hour.

$$
\text { Myth Truth }
$$

7. Before heavy exercise, you should eat a meal high in carbohydrates.

$$
\text { Myth Truth }
$$

\section{How did you do?}

\section{Myth}

Even if you are an active person, you don't need protein or amino acid supplements. These supplements are unnecessary and can be dangerous! Athletes do need more protein than non-athletes. But they easily can get protein from the extra food they eat for the calories they need.

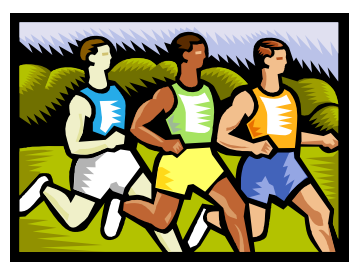

Not only is excess dietary protein not needed, it requires extra work for the body to process. Protein or amino acid supplements may be harmful if taken in excess.

\section{Truth}

The simple carbohydrate glucose is a primary energy source during activity. Even when the body burns fat for energy, we need glucose. By eating a high-carbohydrate diet each day, you will have a ready supply of glucose when you need it.

Your diet will be high in carbohydrates if you follow the MyPyramid eating plan. You can learn about selecting foods for a healthful diet at:

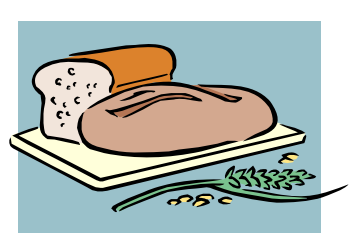
http://mypyramid.gov.

We get carbohydrates in our diets from grains, fruits, vegetables, legumes, and milk. Make these foods the center of your eating plan.

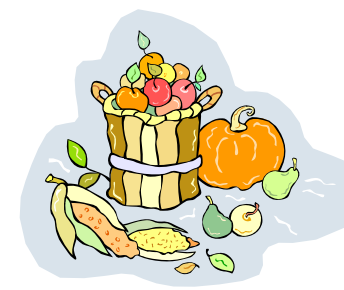

Carbohydrates are stored in the liver and muscles as glycogen. Glycogen is converted to glucose (blood "sugar") when it is needed as fuel for muscle activity. Glycogen stores can provide energy for about two hours of moderate physical activity. Eating a diet with adequate carbohydrates will maintain your glycogen so it will be there when you need it for energy.

\section{Myth}

Vitamins are nutrients that we need in our diets in very small amounts. They help regulate chemical reactions in our bodies. Vitamins are not a direct source of energy, but they help our bodies obtain energy from foods we eat.

Vitamins are widely distributed in foods, but no one food contains all the vitamins we need. This is why MyPyramid recommends that we eat a variety of foods from all five food groups. Eating foods from all of the food groups ensures that we will have an adequate supply of all nutrients in

our diets, including vitamins, needed for fitness and health.

\section{Figure 1}




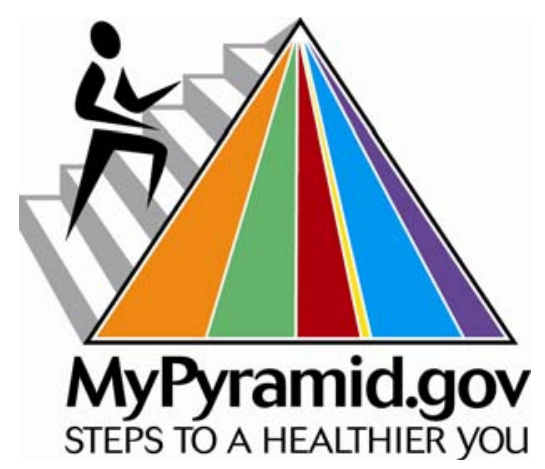

The food group bands represent the five major food groups (plus oil - the thin yellow band). From left to right, they are:

Grains (Make half your grains whole)

Vegetables (Vary your veggies)

Fruits (Focus on fruits)

Milk (Get your calcium-rich foods)

Meat and Beans (Go lean with protein)

People who severely limit their food intake or omit foods from one or more food groups may not get enough vitamins and/or minerals. They may need a multivitamin or mineral supplement to make up for an inadequate intake of these nutrients from foods.

Taking large amounts (megadoses) of vitamins can cause adverse health effects. Even the water soluble vitamins (B complex and C) can cause health problems. Megadoses of vitamins may cause the following:

- $\quad$ irregular heartbeat (niacin),

- $\quad$ nerve degeneration $\left(\mathrm{B}_{6}\right)$,

- diarrhea (vitamin C),

- gastrointestinal upset (vitamin C),

- $\quad$ kidney stones (vitamin C).

Excessive intake of the fat-soluble vitamins A and $\mathrm{D}$ is an even greater health hazard. Since the body can store high levels of these vitamins, toxic levels can build up. This causes illness and sometimes even death. People are more likely to get toxic levels of these vitamins from supplements, rather than food.

\section{Myth}

Reports in the media about chromium often overstate its benefits for performance and weight loss. The truth is that research results are conflicting. Before you take any supplement, there should be clear scientific evidence that it is both effective and safe.

We don't know if chromium supplements improve performance, and there is some concern that chromium picolinate may be harmful. Studies using isolated cells have shown that this form of chromium can cause increased levels of free radicals. Free radicals can cause damage to DNA and may increase risk of cancer.

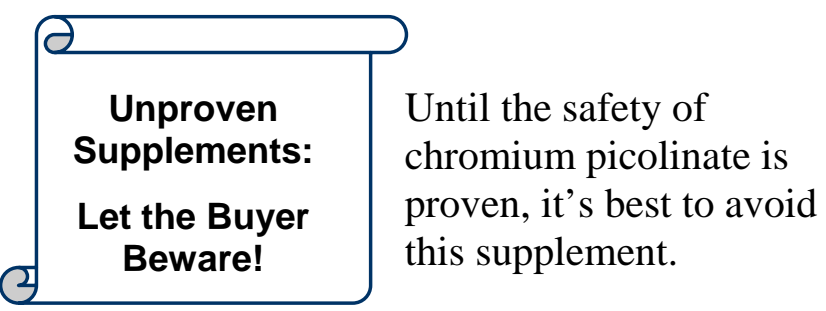

\section{Myth}

Drinking water before, during, and after exercise is essential to prevent dehydration! Dehydration decreases performance and can cause serious harm to the body (see Table 1). Staying well hydrated allows athletes and casual exercisers to perform at their best.

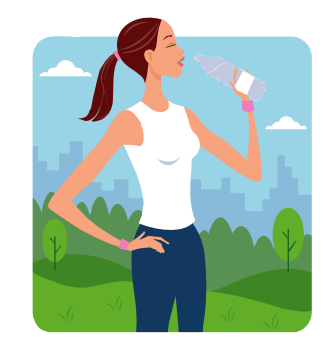

Table 1

\begin{tabular}{|c|l|}
\hline \multicolumn{2}{|c|}{ Effects of Dehydration } \\
\hline Water loss & \multicolumn{1}{c|}{ Symptoms } \\
\hline $1-3 \%$ & Impaired performance \\
\hline $4-6 \%$ & $\begin{array}{l}\text { Chills } \\
\text { Nausea } \\
\text { Rapid pulse } \\
\text { Increased body temperature }\end{array}$ \\
\hline $7-10 \%$ & $\begin{array}{l}\text { Decreased sweat production } \\
\text { Heat stroke } \\
\text { Hallucinations } \\
\text { Loss of coordination and } \\
\text { balance }\end{array}$ \\
\hline
\end{tabular}

Thirst is not a good indicator of fluid needs! You may not feel thirsty until you have lost $1-2 \%$ of your body water. During strenuous exercise, drink one-half to one cup of cool water every 15 to 20 
minutes, whether or not you feel thirsty. Cool water is quickly absorbed and used by your body.

To tell if you're getting enough water, check the color of your urine. It should be light yellow; if it's dark yellow, drink more water! For a strenuous workout, weigh yourself before and after you exercise. For every pound lost, drink two cups of a

Heat stroke results from an uncontrolled increase in body temperature. Symptoms of impending heat stroke include:

- chills

- throbbing pressure in the head

- nausea

- dry skin

If you have any of these

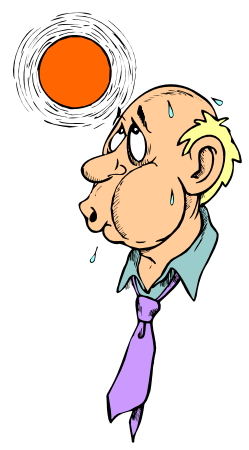
get medical help right away!

\section{Truth}

Water is the best drink for replacing fluid losses during exercise lasting less than one hour. However, during intense exercise lasting longer

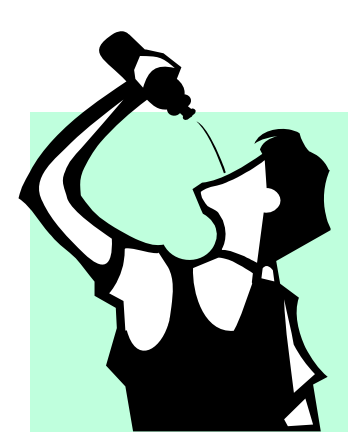
than one hour, you need to sweat and the carbohydrates you burn for energy. Commercial sports drinks supply water, sugar, and electrolytes, including sodium. They promote fluid retention and tend to increase voluntary drinking because of their taste. On the other hand, beverages higher in sugar, including fruit juice, can cause nausea and dehydration. sports drink to replace water and electrolytes. symptoms, stop exercising and replace the sodium lost in

What you eat before exercising can affect your workout. Most people do best if their stomachs are empty when they start to exercise. It takes about three hours for your stomach to empty, so the preexercise meal should be one that's easily digested. Have a small meal high in carbohydrate, moderate in protein, and low in fat. Be sure to include fluids!

The high carbohydrate foods in your meal should contain starch rather than simple sugars. Some foods you might want to try are:

$$
\begin{aligned}
& \checkmark \text { cereal } \\
& \checkmark \text { pancakes } \\
& \checkmark \text { tortillas with reduced fat cheese } \\
& \checkmark \text { pasta with meat sauce }
\end{aligned}
$$

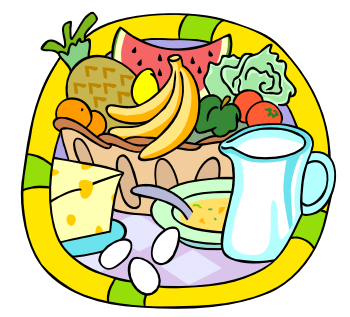

Add low-fat milk, fruit and/or vegetables for a complete meal. Avoid foods that can cause gas, like beans, peas, and vegetables from the cabbage family.

\section{I'm active-Can I eat all the fats and sweets I want?}

No! You may have higher calorie needs than an inactive person, but you want to fuel your body for fitness and health. Eat sparingly foods like candy, mayonnaise, salad dressings, beverages high in sugar like sweet tea and soft drinks, and foods from each of the MyPyramid food groups that are high in fat or sugar. These foods are full of calories, but low in vitamins, minerals, fiber, and protein.

\section{A Note of Caution}

If you have one or more of the following conditions, or if they run in your family, get medical advice before exercising:

\section{Diabetes}

Heart disease

\section{Truth}


$>$ High blood cholesterol

Being active may be a part of your treatment plan for these conditions, but needs to be supervised by your health care team. Have a check-up before changing your activity level.

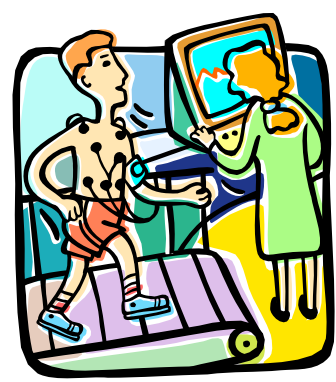

Follow the advice of your doctor and a registered dietitian (RD) regarding your diet and type of

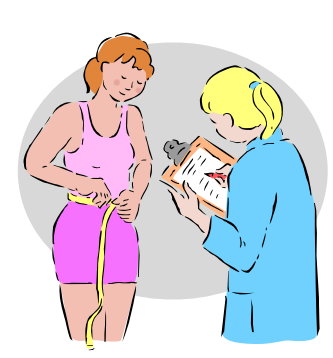
fitness plan. This is especially important if you take medications such as insulin, diuretics, or heart stimulants.

\section{Final Food Facts for Fitness}

* Active people do not require special foods or diet supplements. A generic multivitamin supplement will provide folic acid needed by women of child-bearing age, and other nutrients often consumed in inadequate amounts by young people.

* Active and inactive people need the same basic nutrients. These include vitamins, minerals, carbohydrates, fats, proteins, and water.

* Active people need more water and calories than inactive people.

* It's important to drink fluids before, during, and after

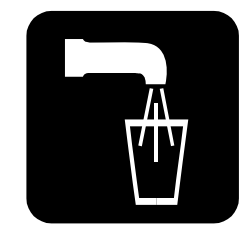
physical activity. Cool fluids are recommended because they are absorbed quickly. Drinking fluids replaces water lost from the body during exercise.

* Sports drinks promote fluid consumption and replace carbohydrates and sodium lost during intense exercise.

* Soft drinks and fruit juices contain enough sugar to cause an unwanted feeling of fullness.
They may cause diarrhea if consumed before exercising.

* Eat a small, easily digested meal, rich in carbohydrates, several hours before exercising vigorously.

\section{Reliable resources on the Internet}

Center for Food Safety \& Applied Nutrition http://www.cfsan.fda.gov

Office of Disease Prevention and Health Promotion http://odphp.osophs.dhhs.gov

WebMD (online health information) http://www.webmd.org

International Food Information Council Foundation http://ific.org

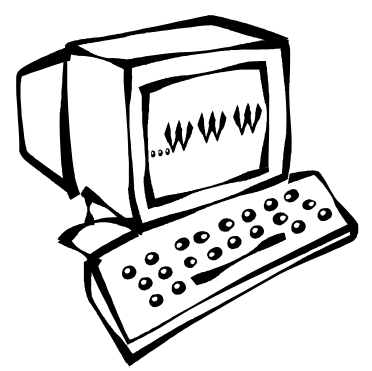

\section{Where can I get more information?}

The Family and Consumer Sciences (FCS) agent at your local Extension office may have written information and nutrition classes for you to attend. Check the blue pages of your telephone book, or find your county office at this Web site: http://www.ifas.ufl.edu/extension/cesmap.htm.

Check your local library for books that are written by qualified health professionals. Magazine articles vary in their reliability; check the credentials of the authors. Articles that attempt to sell a product or service are often biased and not reliable.

Your doctor (MD) or a registered dietitian (RD) can provide reliable information to you. 